\title{
Modelling synergistic interactions between HER2, Sprouty2 and PTEN in driving prostate carcinogenesis
}

\author{
Imran Ahmad ${ }^{1,2,3}$, Meiling Gao ${ }^{1}$, Rachana Patel ${ }^{1}$ and Hing Y Leung ${ }^{1,2,3}$ \\ Asian Journal of Andrology (2013) 15, 323-327; doi:10.1038/aja.2013.40; published online 15 April 2013
}

$\mathrm{P}$ rostate cancer remains a major global health issue and a major cause of morbidity and mortality in men worldwide. Activation of androgen receptor and inactivation of the tumour suppressor gene phosphatase and tensin homologue (PTEN) represent two major events in prostate carcinogenesis. Using a range of clinical resources, in vitro and in vivo models, we explored potential complex interactions among receptor tyrosine kinases (such as HER2/3 and EGFR) and tumour suppressor genes, namely, Sprouty2 (SPRY2) and PTEN. The impacts on their downstream effectors (including PI3K and MAPK) to result in fine regulation of the signalling networks were also considered, which may represent important targets for developing treatment in the context of personalized medicine.

\section{INTRODUCTION}

PCa is now the commonest cancer in men in the developed world, and its incidence continues to rise worldwide as a result of prostate-specific antigen testing and ageing populations. ${ }^{1,2}$ Prostate tumourigenesis involves multistep accumulation of mutations in cancer related genes, promoting the transformation of benign epithelium to locally invasive lesions, which ultimately progress into metastases. However, information on the temporal relationship of individual genes driving prostate carcinogenesis remains scanty. PCa is also highly heterogeneous in terms of tumour morphology and behaviour, thus making it difficult to robustly develop a scheme to capture the different molecular stages of PCa

${ }^{1}$ The Beatson Institute for Cancer Research, Glasgow G61 1BD, UK; ${ }^{2}$ Institute for Cancer Sciences, University of Glasgow, Glasgow G61 1BD, UK and ${ }^{3}$ Department of Urology, NHS Greater Glasgow and Clyde, Glasgow G12 OYN, UK

Correspondence: Professor HY Leung (h.leung@

beatson.gla.ac.uk) development. We believe that distinct aberrant signalling events interact to drive $\mathrm{PCa}$ in a complex synergistic manner, and better understanding of such crosstalks will shed new insight into the underlying mechanisms driving PCa. Figure 1 outlines the key characteristic patterns of genetic and epigenetic abnormalities implicated in prostate carcinogenesis. Several credible candidate genes have been implicated, based on their localisation to regions of allelic loss and their functional properties. However, whether or not these events cooperate in driving PCa development and progression remains to be investigated in more details. In this article, we highlight our recent discoveries on how key signalling molecules may interact in a complex manner to promote tumourigenesis by modelling abnormal cellular signalling events in vitro and in vivo, and consider these findings in the context of developing targeted therapy for future clinical evaluation.

\section{INTERACTION BETWEEN HER2 ACTIVATION AND PTEN LOSS}

Phosphatase and tensin homologue (PTEN) is among the most commonly mutated genes in human cancer, including PCa. Progressive inactivation or loss of PTEN appears to be involved in the development of invasive and metastatic PCa. Transgenic mice with Pten heterozygous deletion in the prostate only develop high-grade prostatic intraepithelial neoplasia (PIN). ${ }^{3}$ The eventual complete loss of PTEN is thought to be a late event and can be considered a critical landmark for progression to aggressive and invasive disease (Figure 1). However, while PCa in the Pten null murine model is invasive, metastatic disease was not observed in our hands, similar to findings of other groups. ${ }^{4}$

Seeking to define potential events that cooperate with PTEN loss to drive prostate carcinogenesis, we tested for clinical correlation between HER2 and PTEN expression in a cohort of 236 PCa patients. For patients with tumours that showed low PTEN and elevated HER2 expression, we observed a reduction in disease-specific survival: cytoplasmic HER2 (median of 7.53 years vs. 2.75 years; $P=0.0004$ ) and membranous HER2 (median of 5.63 years vs. 1.96 years; $P=0.0355)$. Interestingly, similar association was noted for HER3 (cytoplasmic and membranous) expression. ${ }^{5}$ In an attempt to investigate the functional significance of the PTEN/HER2 correlation, we modelled these changes in a transgenic mouse system using the prostate specific promoter, derivatives of the rat probasin $(\mathrm{PB})$ promoter have been engineered into the composite $\mathrm{PB}-\mathrm{Cre} 4$ promoter incorporating two androgen-responsive regions required for androgen receptor $(\mathrm{AR})$ mediated gene expression. ${ }^{6}$ Hence, PB-Cre4 is highly efficient in driving high levels of transgene expression in a prostate-specific manner. ${ }^{7}$ Using PB-Cre4 promoter, we targeted Her2 activation and Pten deletion in the murine epithelium, referred to as PB-Cre4: Pten ${ }^{f l f l} \mathrm{Her}{ }^{\mathrm{KI}}$ thereafter. The control PB-Cre4: Pten ${ }^{f l / f l}$ mice demonstrated a phenotype as previously published, with a slow progression to invasive $\mathrm{PCa}$ ( $>10$ months) and no evidence of metastasis up to 18 months. ${ }^{4}$ However, the double mutant PB-Cre4: ten $^{\text {fllfl }}$ Her $^{K I}$ mice $(n=32)$ developed prostate tumours much faster than the PB-Cre4: Pten ${ }^{f l f l}$ mice (median 465 days vs. 355 days; $P=0.0014$ ) and on autopsy the Pten $^{\text {flfl }} \mathrm{Her}^{\mathrm{KI}}$ prostates were significantly larger in size when compared to tumours from the $P$ ten ${ }^{f l f l}$ mice $(5.2 \mathrm{~g}$ vs. $2.9 \mathrm{~g}$; $P<0.0001)$.

Pten loss-induced cellular senescence (PICS) has been reported to have a key tumour suppressive role in murine prostate cancer, explaining the long tumour latency in this model. ${ }^{4}$ Since we observed acceleration of tumour onset in the PB-Cre4: Pten ${ }^{f l f l} \mathrm{Her} 2^{K I}$ 


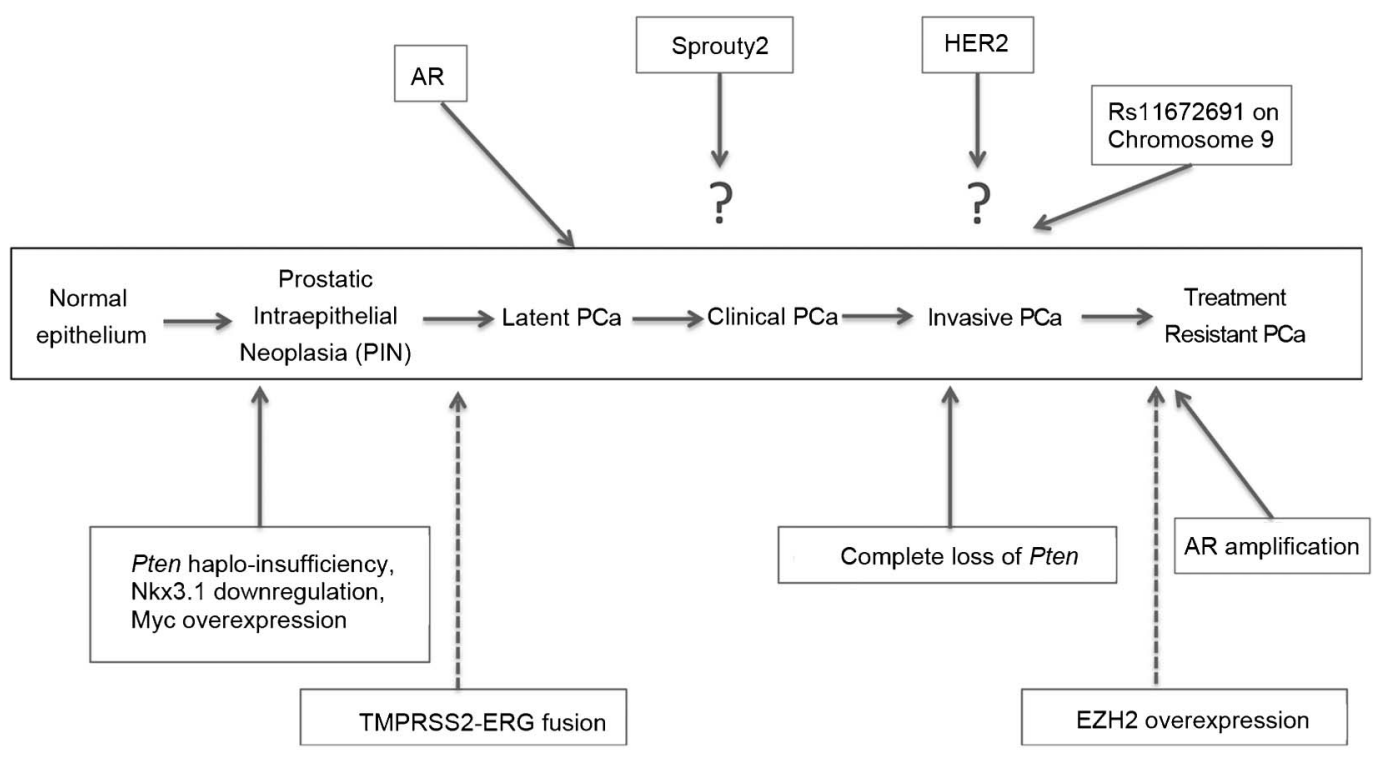

Figure 1 Pathway for human PCa progression. Stages of progression are correlated with loss of specific chromosome regions and candidate tumour suppressor genes (modified from Abate-Shen and Shen). ${ }^{23}$ Dotted lines signify the uncertainty concerning the individual events regarding the exact stage of their involvements during carcinogenesis. PTEN, phosphatase and tensin homologue; AR, androgen receptor.

mice, we hypothesized that Her2 activation may modify this pathway. In contrast to AKT activation seen upon PTEN loss alone, heregulin (a soluble activating ligand for HER2-HER3) potently induced the MAPK pathway. Treatment with a specific PI3K or MEK inhibitor (LY294002 and PD184352, respectively) demonstrated a strong reciprocal feedback regulation of PI3K-AKT and MEK-ERK signalling cascades to control proliferation. To test whether the observed HER2-driven MAPK activation was mechanistically sufficient to overcome PICS, we treated 12-month-old tumour bearing PB-Cre4: Pten ${ }^{f l f l}$ Her $2^{K I}$ mice with the MEK1/2 inhibitor PD184352. After just 7 days of treatment, we observed a significant difference in tumour bulk between the treatment and vehicle group (median difference in tumour weight of $1.1 \mathrm{~g}$; $P=0.04)$. There was corresponding reduced proliferation, increased apoptosis and upregulation of markers indicative of cellular senescence in the tumours in the treated group.

In summary, our data suggest that Her2 activation drives MEK-MAPK activation in vitro and in vivo to attenuate growth arrest and senescence, while MEK inhibition restored a PICS-like phenotype to the $P B$ Cre4: Pten ${ }^{\text {fl/fl }} \mathrm{Her}^{\mathrm{KI}}$ tumours. Hence, stratifying patients according to their tumour PTEN and HER2 status might help in predicting the responsiveness to targeted therapy with MEK/ERK inhibition. For instance, our data presented here suggest MEK inhibitors to be effective targeted therapy in $\mathrm{PCa}$ tumours with high HER2 levels and low levels of PTEN (Figure 2).

\section{SPRY2 LOSS PROMOTES HER2- MEDIATED EFFECTS ON PROSTATE CARCINOGENESIS}

EGFR signalling forms a complex signalling network with positive and negative regulators. Sprouty2 (SPRY2) is a key regulator of multiple receptor tyrosine kinase signalling pathways, and is frequently inactivated in human malignancies. ${ }^{8-10}$ Emerging evidence increasingly supports Sprouty2 (SPRY2) as a key tumour suppressor gene. Given the impact of SPRY2 on the function of multiple receptor tyrosine kinases, we examined the expression status of SPRY2 and all four members of the HER/ErbB family (namely, HER1-4) in a cohort of clinical PCa. ${ }^{11}$ Among patients with reduced SPRY2 expression, overexpression of cytoplasmic HER2 significantly conferred shorter patient survival $(P=0.014)$. The other HER members did not show any association with patient outcome. Reassuringly, similar results were obtained from the cBio Genomic portal; alteration in SPRY2 gene significantly reduced the disease-free period in PCa patients $(P=0.000056)$.

To examine the functional significance of SPRY2 loss in prostate carcinogenesis, we generated stable SPRY2 knockdown (KD) clones of PCa cell lines. Through a variety of stimulation experiments with EGF and heregulin, we demonstrated that loss of SPRY2 promotes rapid internalisation and sustained accumulation of intracellular EGFR and HER2 in response to EGF stimu- lation in a PTEN-dependent manner. Using in vitro functional assays, we observed significantly enhanced EGF-mediated proliferation and invasion in SPRY2 KD cells, which was at least in part due to sustained EGFR/HER2 signalling in a PTEN-dependent manner. Indeed, knocking down PTEN expression in SPRY2 KD cancer cells impaired EGF-induced invasion and abolished the observed upregulation of proliferation. Taken together, we demonstrated that loss of SPRY2 cooperates with HER2 signalling to enhance proliferation and invasion in a PTEN-dependent manner. This would suggest that SPRY2 loss and HER2 activation are particularly important in prostate carcinogenesis in early to local invasive disease prior to the development of metastatic lesions where PTEN is often completely inactivated or lost.

We further examined downstream signalling following EGF stimulation in PTEN proficient SPRY2 KD cancer cells using a human phospho-kinase array ${ }^{11}$ and found that EGFmediated activation of both PI3K/AKT and MAPK pathways were enhanced in SPRY2 KD PCa cells; this observation was also confirmed independently by Western blot analysis. The relative significance of PI3K/AKT and MAPK in SPRY2 KD cells were then tested. SPRY2 KD DU145 cells were therefore treated with a PI3K inhibitor (LY294002, $10 \mu \mathrm{mol}$ $\left.1^{-1}\right)$, which significantly reduced proliferation and invasion $(P=0.001$ and $P=0.002$, respectively). In contrast, treatment with the MEK inhibitor (PD098059, $10 \mu \mathrm{mol} \mathrm{l^{-1 }}$ ) did not impact on proliferation or invasion. 


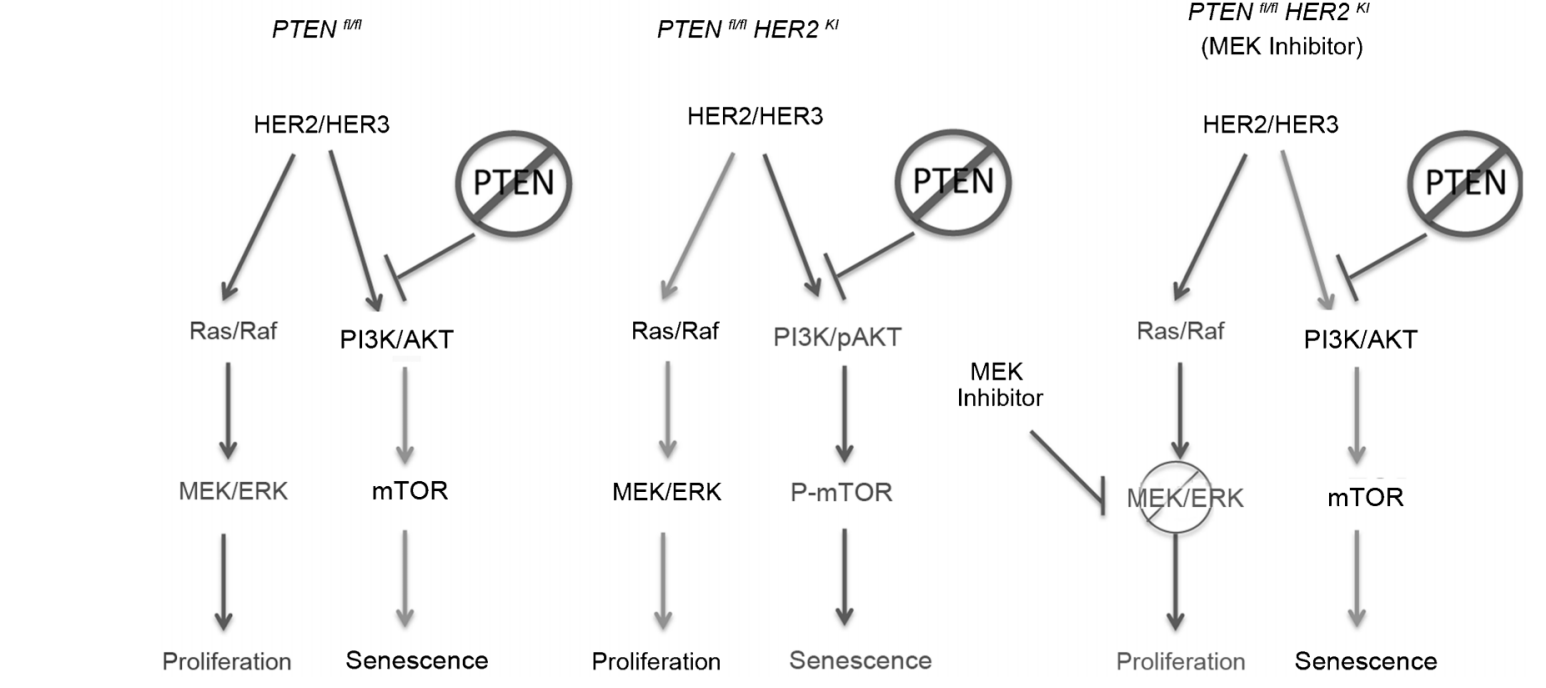

PTEN ${ }^{\text {IIII }}$ HER2 ${ }^{\mathrm{K} I}$

PTEN ${ }^{\text {H/n HER }}{ }^{\mathrm{KI}}$

(MEK Inhibitor)

Figure 2 Mechanism of tumorigenesis in PB-Cre4: Pten ${ }^{f l f l} H_{e r}{ }^{K I}$ mice. PB-Cre4: Pten ${ }^{f l / f l}$ tumours demonstrate upregulation of the PI3K pathway, which upregulates p53 and leads to a senescent phenotype. In the PB-Cre4: Pten ${ }^{f l / f l} H e r 2^{K I}$ tumours, upregulation of the MAPK 'outweighs' the PI3K upregulation, leading to a proliferative phenotype. When these mice are treated with a MEK1/2 inhibitor, the proliferative phenotype via the MAPK pathway is suppressed and the senesencent phenotype (via the PI3K pathway) predominates. PTEN, phosphatase and tensin homologue.

Immuno-fluorescence studies revealed retention of EGFR and HER2 at high levels within the early endosomes. In addition, suppression of clathrin-mediated endocytosis by dynasore (a cell permeable inhibitor of dynamin) dramatically block EGFR endocytosis, and the observed p-AKT activation in SPRY2 KD cells was abolished despite EGF stimulation, thus confirming the importance of endosomal EGFR in promoting PI3K/AKT signalling. p38 is a known regulatory kinase of EGFR at Ser-1046/1047, and is critical for EGFR internalisation ${ }^{12}$ with a pro-survival effect. $^{13}$ Elevated phospho-p38 level in SPRY2 KD cells was confirmed. In addition, both pharmacological inhibition p38 function and siRNA-mediated knockdown of p38 expression abolished activation of AKT induced by EGF, by significantly suppressing EGFR internalisation and thus reducing both cellular growth and invasion. Collectively, these results indicate that SPRY2 loss results in activation of PI3K and its downstream effector p38 to promote EGFR trafficking and signalling, via a positive feedback loop (Figure 3). ${ }^{11}$

We next tested if combined in vivo haploinsufficiency of Spry2 and Pten may result in enhanced HER signalling. A Pten ${ }^{f l /+}$ Spry $2^{+/-}$mouse was generated and the prostate tissue was harvested from mice at 12 months. Consistent with previous reports, ${ }^{14}$ Pten $^{f l /+}$ mice developed the pre-malignant PIN lesions in their prostate glands, while combined haplo-insufficiency of Spry2 and Pten resulted in aggressive prostate tumours.
As we hypothesized, HER2 expression was significantly upregulated in Pten ${ }^{f / /+}$ Spry $^{+/-} \mathrm{PCa}$, along with enhanced AKT activation. Therefore, Pten ${ }^{f l+}$ Spry2 $^{+/-}$mice harbouring prostate tumours were treated with a PI3K inhibitor, following which the prostatic tumour showed reduced proliferation and reduced $\mathrm{p}$ AKT levels. Consistent with our model, both EGFR and HER2 expression were significantly reduced. Considering the lymph node status as an indicator of the aggressiveness of the tumours upon PI3K inhibition, three of four $\mathrm{Pten}^{f l /+}$ Spry $2^{+/-}$mice treated with vehicle control developed metastatic nodal lesions. In contrast, none of the four PI3K-treated animals developed metastatic nodal disease $(P=0.025)$. In summary, enhanced PI3K/AKT signalling significantly contributes to prostate carcinogenesis driven by SPRY2 and PTEN loss.

\section{DISCUSSION}

The overall purpose of our studies was to gain new insight into how complex interaction between signalling networks may promote $\mathrm{PCa}$ progression and to begin developing meaningful preclinical model systems to assess new therapies. Collectively, we confirmed an important role for PTEN along with interesting synergism with either HER2 activation or Sprouty2 loss. Both of these interactions have identified certain tumour signalling profiles that may be particularly suitable for specific targeted therapy. These data warrant further formal therapeutic assessment in preclinical in vivo models.
In addition, our data add to the mounting evidence that complex multiple signalling cascade interacts to promote tumourigenesis including treatment resistance. Table 1 summarizes the key relevant events.

In our first study, we demonstrated that patients with PCa that have low PTEN and accompanying HER2/3 overexpression have a relatively poor prognosis. Neither factor in isolation alters survival in human PCa. In the mouse, presence of both mutations cooperates to drive prostate carcinogenesis by overcoming growth arrest/senescence induced by PTEN loss. Importantly, treatment with a MEK inhibitor appears to negate the effects of activated HER2, restoring PICS phenotype. A recent large-scale sequencing study found PTEN inactivating mutations in $4 \%$ of primary and $42 \%$ of metastatic prostate tumours. When the authors examined the entire PI3K pathway, they found deregulation in $42 \%$ of all primary tumours and $100 \%$ of all metastases. ${ }^{18}$ Therefore, within human PCa, deregulation of PI3 kinase signalling appears essential for $\mathrm{PCa}$ progression. Murine studies have shown that Pten heterozygosity can act to promote tumour initiation and progression. ${ }^{19}$ However, complete PTEN loss can provoke a p53dependent cellular senescence program and thus, tumourigenesis is protracted in $P B$ Cre4: Pten ${ }^{f l / f l}$ animals, a finding that we confirmed. ${ }^{4}$ This suggests that while Pten haplo-insufficency can initiate PCa, selection for loss of the remaining allele of Pten will not occur until later PCa progression when other 
Table 1 Summary of selected recent data on prostate carcinogenesis based on transgenic mouse studies

\begin{tabular}{|c|c|c|}
\hline Author & Data & Reference \\
\hline Hong Wu & $\begin{array}{l}\text { Demonstrated that castration-resistant growth is an intrinsic property of Pten null PCa cells, independent of cancer development } \\
\text { stage. PTEN loss suppresses androgen-responsive gene expressions by modulating AR transcription factor activity. In the } \\
\text { mouse, conditional deletion of } A r \text { in the epithelium promotes the proliferation of Pten null cancer cells, partially, by } \\
\text { downregulating the androgen-responsive gene Fkbp5 and preventing PHLPP-mediated AKT inhibition }\end{array}$ & 15 \\
\hline $\begin{array}{l}\text { Neal Rosen and Charles } \\
\text { Sawyers }\end{array}$ & $\begin{array}{l}\text { Demonstrated that AR transcriptional output is decreased in human and murine tumours with PTEN deletion and that PI3K } \\
\text { pathway inhibition activates AR signalling by relieving feedback inhibition of HER kinases. Similarly, AR inhibition activates AKT } \\
\text { signalling by reducing levels of the AKT phosphatase PHLPP. Thus, these two oncogenic pathways were found to cross-regulate } \\
\text { each other by reciprocal feedback. Inhibition of one activates the other, thereby maintaining tumour cell survival. However, } \\
\text { combined pharmacologic inhibition of PI3K and AR signalling caused near-complete prostate cancer regressions in a Pten- } \\
\text { deficient murine PCa model and in human PCa xenografts, indicating that both pathways coordinately support survival }\end{array}$ & 16 \\
\hline William Sellers & $\begin{array}{l}\text { Showed that activated AKT1 only induced PIN without progression to invasive cancer in the murine prostate. In luminal epithelial } \\
\text { cells of Akt-driven PIN, they have shown the concomitant induction of p27(Kip1) and senescence. Genetic ablation of p27(Kip1) } \\
\text { led to downregulation of senescence markers and progression to cancer. In humans, p27(Kip1) and senescence markers were } \\
\text { elevated in PIN not associated with PCa, but were decreased or absent, respectively, in cancer-associated PIN and in PCa. } \\
\text { Importantly, p27(Kip1) upregulation in mouse and human in situ lesions did not depend upon mTOR or Akt activation, but was } \\
\text { instead specifically associated with alterations in cell polarity, architecture and adhesion molecules }\end{array}$ & 17 \\
\hline
\end{tabular}

Abbreviations: AR, androgen receptor; PCa, prostate cancer; PIN, prostatic intraepithelial neoplasia; PTEN, phosphatase and tensin homologue.

signalling pathways are deregulated which block PICS. ${ }^{4}$ From our studies, we postulate that activation of the HER2 signalling is one such pathway in human cancer that overcomes this, leading to an aggressive $\mathrm{PCa}$ phenotype. Mechanistically in our study, we suggest that Her2-mediated activation of the MAPK pathways overcomes PICS to drive tumour progression.

Currently, multiple MEK inhibitors are in clinical trials against a variety of advanced cancers. ${ }^{20}$ It is still uncertain which tumour groups are more likely to respond to MEK1/2 inhibition. Thus, selection of appropriate patient populations based on genetic lesions or validated biochemical markers will be critical for future clinical trial evaluation. Indeed, the complex nature of advanced cancers suggests that MEK1/2 inhibitors be required in combination with other targeted agents/cytotoxic drugs. Our observations suggest that the combination of MEK1/2 and PI3K inhibition in cancers that harbour concurrent activating mutations in these signalling pathways may be particularly efficacious.

Synergism resulting from interaction between combined haplo-insufficiency of SPRY2 and PTEN in driving prostate tumourigenesis is highly novel. We uncovered a critical feedback system, whereby SPRY2 loss leads to PI3K/AKT function coupled to activation of p38 MAPK in driving EGFR trafficking and its sustained intracellular localisation. The observed PI3K/AKT activation is PTENdependent and is at least in part mediated by enhanced phosphorylation of PTEN. ${ }^{21}$ Our in vivo studies on a mouse prostate model and clinical PCa specimens provided additional evidence for a key role of PI3K/ AKT in prostate carcinogenesis, particularly in the context of SPRY2 loss. Hence, our hypothesis is that PCa with SPRY2 loss, partial inactivation of PTEN and HER2 expression are particularly susceptible to PI3K/AKT targeted therapy. In addition, further reduction or complete loss of PTEN along with impaired SPRY2 expression

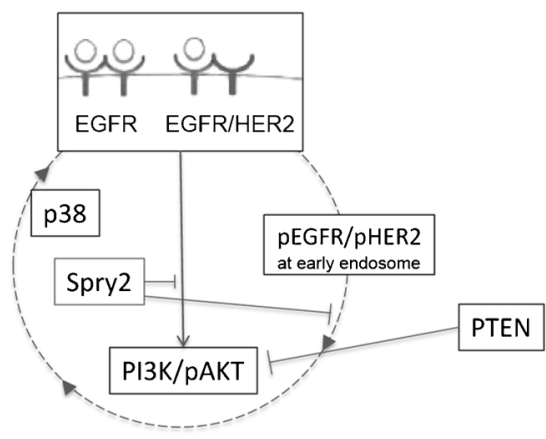

Figure 3 Enhanced recycling of EGFR/HER2 upon Sprouty2 loss in a PTEN-dependent manner via sequential activation of PI3K/AKT and p38. PTEN, phosphatase and tensin homologue. resulted in RTK (EGFR and HER2) degradation, thus rendering them insensitive to therapies targeting these receptors.

Recent evidence suggested combinatory targeted therapies could produce enhanced treatment efficacy. For instance, MAPK and $\mathrm{PI} 3 \mathrm{~K} / \mathrm{AKT}$ are simultaneously and synergistically activated in a $\mathrm{Pten}^{+/-}$mouse prostate model. ${ }^{22}$ Our genetically modified $\left(\right.$ Pten $^{+/-}$Spry $\left.^{+/-}\right)$mouse model showed evidence of PI3K/AKT hyperactivation, without MAPK activation, in the prostate and enhanced capability to develop metastatic nodal disease by 12 months. Significantly, PI3K inhibition exerted a dramatic inhibitory effect on the development of nodal metastasis. Taken together, the selective activation of PI3K/AKT associated with SPRY2 loss in the context of PTEN insufficiency is important in prostate carcinogenesis.

Given the time and resources required to apply genetically modified mouse models (GEMMs) in the evaluation of novel therapies, refinement and new applications of these resources are required. One possibility is to prepare xenografts from the prostate tumours developed from the relevant genetic backgrounds, including subcutaneous, subrenal capsule or orthotopic implantation. Orthografts of GEMM-derived PCa tumours are particularly appealing as it incorporates the molecular signature of the GEMMderived tumours while maintaining the in vivo microtumour environment required for both local and metastatic disease progression. Future efforts as outlined by Figure 4 will be useful as a strategy to assess new drugs as well as to probe the molecular basis of tumour progression. 


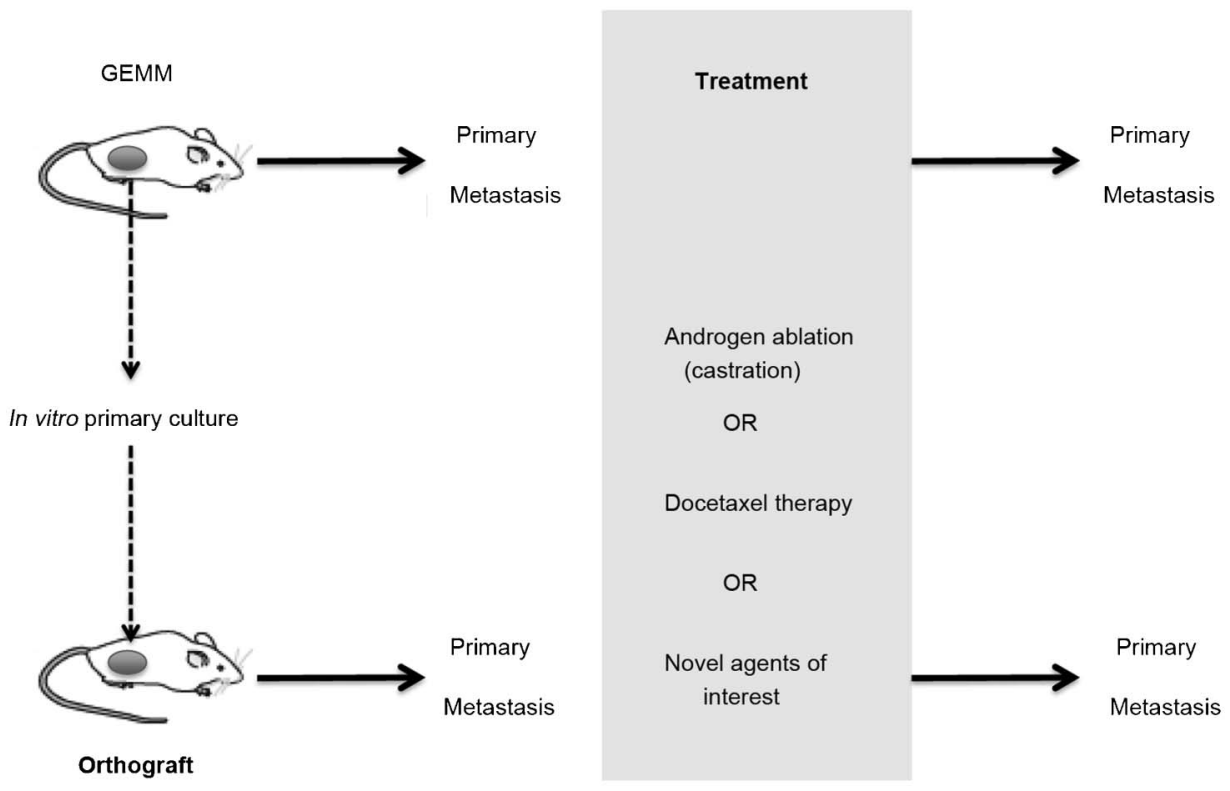

Figure 4 Methods of utilisation of tumours generated from GEMMs. Orthografts derived from GEMM tumours can be useful in vivo models to study both primary and metastatic tumour growth and their responses to treatment. GEMM, genetically modified mouse model.

\section{CONCLUSION}

Complex interactions among receptor tyrosine kinases (such as HER2/3 and EGFR) and tumour suppressor genes (including SPRY2 and PTEN) and their downstream effectors (PI3K and MAPK) exhibit fine regulation of the signalling networks to involve distinct molecules, which may represent important targets for developing treatment in the context of personalized medicine.

\section{COMPETING FINANCIAL INTERESTS}

The authors declare no competing financial interests.

\section{ACKNOWLEDGMENTS}

Research work in the Prostate Research Group, Beatson Institute for Cancer Research, is funded by Cancer Research UK, Prostate Cancer UK, Wellcome Trust, Medical Research Council and the Greater Glasgow and Clyde NHS Endowment. The authors thank collaborators (past and present) involved in studies reviewed in this article.

1 Schroder FH, Hugosson J, Roobol MJ, Tammela TL, Ciatto $S$ et al. Screening and prostate-cancer mortality in a randomized European study. N Engl J Med 2009; 360: 1320-8.

2 Andriole GL, Crawford ED, Grubb RL 3rd, Buys SS, Chia $\mathrm{D}$ et al. Mortality results from a randomized prostate-cancer screening trial. N Engl J Med 2009; 360: 1310-9
3 Mellinghoff IK, Vivanco I, Kwon A, Tran C, Wongvipat J et al. HER2/neu kinase-dependent modulation of androgen receptor function through effects on DNA binding and stability. Cancer Cell 2004; 6: 517-27.

4 Chen Z, Trotman LC, Shaffer D, Lin HK, Dotan ZA et al. Crucial role of p53-dependent cellular senescence in suppression of Pten-deficient tumorigenesis. Nature 2005; 436: 725-30.

5 Ahmad I, Patel R, Singh LB, Nixon C, Seywright M et al. HER2 overcomes PTEN (loss)-induced senescence to cause aggressive prostate cancer. Proc Natl Acad Sci USA 2011; 108: 16392-7.

6 Kasper S, Rennie PS, Bruchovsky N, Sheppard PC, Cheng $\mathrm{H}$ et al. Cooperative binding of androgen receptors to two DNA sequences is required for androgen induction of the probasin gene. $J$ Biol Chem 1994; 269: 31763-9.

7 Wu X, Wu J, Huang J, Powell WC, Zhang J et al. Generation of a prostate epithelial cell-specific Cre transgenic mouse model for tissue-specific gene ablation. Mech Dev 2001; 101: 61-9.

8 Weber $\mathrm{C}$, Fraemohs L, Dejana E. The role of junctional adhesion molecules in vascular inflammation. Na Rev Immunol 2007; 7: 467-77.

9 Woodfin A, Reichel CA, Khandoga A, Corada M, Voisin $\mathrm{MB}$ et al. JAM-A mediates neutrophil transmigration in a stimulus-specific manner in vivo: evidence for sequential roles for JAM-A and PECAM-1 in neutrophil transmigration. Blood 2007; 110: 1848 56.

10 Faratian D, Sims AH, Mullen P, Kay C, Um I et al. Sprouty 2 is an independent prognostic factor in breast cancer and may be useful in stratifying patients for trastuzumab therapy. PLOS ONE 2011; 6: e23772.

11 Gao M, Patel R, Ahmad I, Fleming J, Edwards J et al. SPRY2 loss enhances ErbB trafficking and PI3K/AKT signalling to drive human and mouse prostate carcinogenesis. EMBO Mol Med 2012; 4: 776-90.

12 Nishimura M, Shin MS, Singhirunnusorn P, Suzuki S, Kawanishi $\mathrm{M}$ et al. TAK1-mediated serine/threonine phosphorylation of epidermal growth factor receptor via p38/extracellular signal-regulated kinase: NF$\kappa \mathrm{B}$-independent survival pathways in tumor necrosis factor alpha signaling. Mol Cell Biol2009; 29: 5529 39

13 Zwang Y, Yarden Y. p38 MAP kinase mediates stressinduced internalization of EGFR: implications for cancer chemotherapy. EMBO J 2006; 25: 4195206.

14 Shen MM, Abate-Shen C. Molecular genetics of prostate cancer: new prospects for old challenges. Genes Dev 2010; 24: 1967-2000.

15 Mulholland DJ, Tran LM, Li Y, Cai H, Morim A et al. Cell autonomous role of PTEN in regulating castrationresistant prostate cancer growth. Cancer Cell 2011; 19: 792-804

16 Carver BS, Chapinski C, Wongvipat J, Hieronymus H Chen $\mathrm{Y}$ et al. Reciprocal feedback regulation of PI3K and androgen receptor signaling in PTEN-deficient prostate cancer. Cancer Cell 2011; 19: 575-86.

17 Majumder PK, Grisanzio C, O'Connell F, Barry M, Brito JM et al. A prostatic intraepithelial neoplasiadependent p27 Kip1 checkpoint induces senescence and inhibits cell proliferation and cancer progression. Cancer Cell 2008; 14: 146-55.

18 Taylor BS, Schultz N, Hieronymus H, Gopalan A, Xiao $Y$ et al. Integrative genomic profiling of human prostate cancer. Cancer Cell 2010; 18: 11-22.

19 Di Cristofano A, Pesce B, Cordon-Cardo C, Pandolfi PP. Pten is essential for embryonic development and tumour suppression. Nat Genet 1998; 19: 348-55.

20 Fremin C, Meloche S. From basic research to clinical development of MEK $1 / 2$ inhibitors for cancer therapy. J Hematol Oncol 2010; 3: 8 .

21 Edwin F, Singh R, Endersby R, Baker SJ, Patel TB. The tumor suppressor PTEN is necessary for human Sprouty 2-mediated inhibition of cell proliferation. J Biol Chem 2006; 281: 4816-22.

22 Gumerlock PH, Chi SG, Shi XB, Voeller HJ, Jacobson JW et al. p53 abnormalities in primary prostate cancer: single-strand conformation polymorphism analysis of complementary DNA in comparison with genomic DNA. The Cooperative Prostate Network. J Natl Cancer Inst 1997; 89: 66-71.

23 Abate-Shen C, Shen MM. Molecular genetics of prostate cancer. Genes Dev 2000; 14: 2410-34. 